\title{
Sensory Incapacitants
}

\section{Introduction}

Sensory incapacitants such as riot control agents are irritants characterised by a very low toxicity, rapid onset and a short duration of action. In general, these agents have a very wide margin of safety. Orthochlorobenzylidene malononitrile (CS) is the most commonly used sensory incapacitant for riot control purposes. Chloracetophenone (CN) is also used in some countries for this purpose in spite of its higher toxicity. Dibenzoxazepine (CR) is a more modern irritant, but there is little experience of its use.

Arsenical smokes (sternutators) have in the past been used on the battlefield. Apart from their lachrymatory action they also provoke other effects such as bronchoconstriction and emesis; they are sometimes referred to as vomiting agents.

\section{CS (ORTHOCHLOROBENZYLIDENE MALONONITRILE)}

CS is used as a riot control agent in many countries. It is also commonly used as a training agent for simulation of chemical warfare agent exposure and in the testing of respirators performance. The limit of the threshold of human detection (slight irritation of the nasal passages) of CS is approximately $0.004 \mathrm{mg} \cdot \mathrm{m}^{-3}$. The minimal irritant concentration ranges from 0.1 to 1.0 $\mathrm{mg} \cdot \mathrm{m}^{-3}$ and intolerable signs and symptoms of exposure occur at concentrations of 4.0 to $10.0 \mathrm{mg} . \mathrm{m}^{-3}$. The estimated humans lethal dose of CS is between 25000 and 150000 $\mathrm{mg} . \mathrm{min} . \mathrm{m}^{-3}$ giving a safety ratio of the order of 25000 to 1500000 .

\section{Properties}

Because of its potent irritant effects and its lower toxicity, CS has superseded $\mathrm{CN}$ as the principal riot control agent. It is a white crystalline solid substance. Solubility is very poor in water, moderate in alcohol and good in acetone, chloroform, methylene dichloride, ethylacetate and benzene. CS is unstable in aqueous solution. CS is usually dispersed as an aerosol generated pyrotechnically, but it can also be disseminated by spraying a solution of CS in a suitable solvent (Figure 27), and as a very fine powder (micronised CS).

Although the smoke is non-persistent, CS may remain on rough surfaces (e.g., clothes) from which it is released only slowly. At least
$1 \mathrm{~h}$ of aeration is necessary to cleanse such materials after exposure.

\section{Detection}

No field detectors for CS exist. The CS smoke is white at the point of release and for several seconds after release. Exposure is associated with a pepper-like odour, and the onset of intense eye irritation, dyspnoea, coughing and rhinorrhoea.

\section{Protection}

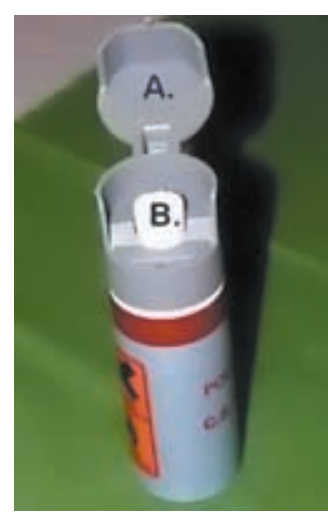

Fig 27.CS spray canister tective equipment will Britain.

provide complete protection. Protection against field concentrations of CS is provided by the respirator and ordinary field clothing secured at the neck, wrists and ankles. Individuals who handle CS should wear rubber gloves, hood, rubber boots, rubber apron and respirator and secure their field clothing at the neck, wrists and ankles.

\section{Decontamination}

Exposed persons should move to fresh air, separate from other contaminated individuals, face into the wind with eyes open and breathe deeply. Contaminated eyes and skin should be flushed with copious amounts of water. Following exposure, clothing and individual equipment should be inspected for residue. If a residue is found, individuals should change and wash their clothing to protect themselves and other unmasked persons.

\section{Mechanism of Action}

Lachrymators act on the nerve endings, the cornea, mucous membranes and the skin. The reaction is very rapid.

\section{Signs and Symptoms}

Exposure to CS causes the following symptoms:

Eyes. Symptoms include a violent burning sensation, conjunctivitis (lasting up to 30 min), erythema of the eyelids (lasting about an hour) blepharospasm, violent lachrymation (over 10-15 min) and photophobia.

Respiratory Tract. The first symptom is a burning sensation in the throat, developing 
into pain and extending to the trachea and bronchi. At a later stage, a sensation of suffocation may occur, often accompanied by fear. In addition, a burning sensation in the nose, rhinorrhoea, erythema of the nasal mucous membranes and sometimes mild epistaxis occur. The sense of taste is often distorted for some hours after exposure. Nausea, diarrhoea and headache have been observed. Sneezing occurs after mild exposure and may be persistent. Many exposed people have reported fatigue for some hours afterwards. Coughing, choking, retching and (rarely) vomiting occur after exposure. Exposure to high concentrations of CS may result in pulmonary oedema.

Skin. A burning sensation occurs, especially in moist areas, but soon disappears. This burning sensation may recur some hours later, often while washing the area. Prolonged exposure to large amounts (e.g. when handling CS in bulk) can cause erythema and vesicle formation. Prolonged exposure (continuous or intermittent) to high concentrations may result in a cumulative effect, when combined with high temperatures and humidity. Sensitivity to CS may be provoked.

\section{First Aid}

In practically all cases it is sufficient to remove the casualty into fresh air; the symptoms will soon disappear. Clothing should be changed. If symptoms persist, the eyes, mouth and skin may be washed with water (and with soap in the case of the skin). Oil based lotions should not be used. Skin decontaminants containing bleach should not be used, but should be reserved for more dangerous contamination (e.g. vesicants or nerve agents); bleach reacts with CS to form a combination which is more irritant to the skin than CS alone.

\section{Treatment}

Eyes. Ordinarily the eye effects are selflimiting and require no treatment. If large particles or droplets of agent have entered the eye, treatment for corrosive materials may be required. Prompt irrigation with copious amounts of water is the best treatment for solid CS in the eye. After complete decontamination, corticosteroid eye preparations may be used in consultation with an ophthalmologist.

Skin. Early erythema and stinging sensation (up to $1 \mathrm{~h}$ ) especially in warm moist skin areas, are usually transient and require no treatment. Inflammation and blistering similar to sunburn may occur after heavy or prolonged exposure, especially in fair skin. Acute contact dermatitis should be managed initially in the same way as any other acute dermatitis. Corticosteroid cream or calamine lotion may be applied to treat existing dermatitis or to limit delayed erythema. Significant pruritus can be treated with calamine lotion or corticosteroid preparations. If blisters develop, they should be treated as any other second degree burn. Secondary infection is treated with appropriate antibiotics.

Respiratory Tract. In the rare event of pulmonary effects from massive exposure, evacuation is required. Management is the same as that for lung damaging agents.

\section{Course and Prognosis}

Most personnel affected by riot control agents require no medical attention and casualties are rare.

\section{CR (DIBENZOXAZEPINE)}

$\mathrm{CR}$ is similar in its effects to CS, but the minimum effective concentration is lower and the $\mathrm{LCt}^{50}$ is higher. Symptomatology and treatment are similar to those of CS.

It is a pale yellow crystalline solid which melts at $73^{\circ} \mathrm{C}$ and is stable in organic solutions. It has limited solubility in water and is not hydrolysed in aqueous solutions. It has a pepper-like odour. CR is an ocular irritant at concentrations down to $0.0025 \%$ or even lower. CR differs from CS in being less toxic when inhaled but skin effects are more pronounced. It is more persistent in the environment and on clothing.

\section{CN (CHLORACETOPHENONE)}

$\mathrm{CN}$ is a riot control agent and as a training agent is now superceded by CS, the latter being much less toxic. However, it is still in use by police in some countries.

\section{Properties}

$\mathrm{CN}$ is a clear yellowish brown solid which melts at around $54^{\circ} \mathrm{C}$. Although poorly soluble in water, it freely dissolves in a wide range of organic solvents. When generated pyrotechnically, it is said to have a faint odour reminiscent of apple blossom. The threshold concentration for human detection is approximately $0.03 \mathrm{mg} \cdot \mathrm{m}^{-3}$ and the effective arassing concentration in the range of 2 to $4 \mathrm{mg}$ minimal irritant concentration is $0.3 \mathrm{mg} \cdot \mathrm{m}^{-3}$. It has been estimated from experimental data that the LCt50 for man is 7000 to $14000 \mathrm{mg} . \mathrm{min} \cdot \mathrm{m}^{-3}$, but inhalation of $350 \mathrm{mg} \cdot \mathrm{m}^{-3}$ for $5 \mathrm{~min}$ may be dangerous. The $\mathrm{ICt} 50$ is 20 to $40 \mathrm{mg} \cdot \mathrm{min} \cdot \mathrm{m}^{-3}$. CN is more toxic than CS.

\section{Mode of Action and Toxic Effects}

The mode of action is similar to that of CS; $\mathrm{CN}$ causes stimulation of sensory nerve endings.

\section{Signs and Symptoms}

Exposure to $\mathrm{CN}$ primarily affects the eyes, producing a burning sensation, lachrymation, inflammation and oedema of the eyelids, blepharospasm, photophobia. At high concentrations, it produces temporary blindness. The most severe of these 
symptoms is reached in a few minutes and then gradually decreases. After about 1 or 2 $\mathrm{h}$, all symptoms disappear. High concentrations can cause irritation of the upper respiratory tract, inflammation of the skin with vesicle formation, visual impairment and pulmonary oedema. Drops or splashes in the eye may cause corrosive burns, corneal opacity and even permanent visual impairment. Drops or splashes on the skin may cause papulovesicular dermatitis and superficial skin burns. Ingestion of food or water contaminated with $\mathrm{CN}$ causes nausea, vomiting and diarrhoea.

\section{First Aid}

After exposure, ill effects are adequately neutralised by allowing fresh air to blow into the open eyes. If necessary, the eyes may be washed with copious amounts of water. The eyes should never be rubbed as mechanical injury may complicate the chemical effect. Patients suffering from temporary blindness should be reassured; permanent blindness from exposure to aerosol has never been observed, even at very high concentrations.

\section{Further Reading}

Ballantine B. "Riot Control Agents." Biochemical and Health Aspects of the Use of Chemicals in Civil Disturbances. Special Article, pp 7-41.

Ballantine B, Callaway S. "The Toxicology and Pathology of Animals Exposed to O-Chlorobenzylidene Malononitrile (CS)." Medicine, Science and Law, Vol 12, 34-65, 1962.

Cucinell SA, Swentzel KC. et al. "Biochemical Interactions and Chemical Fate of Riot Control Agents." Fed. Proc. Vol 30;1:Jan-Feb 1971.

Hellreich A, Mershon MM. et al. "An Evaluation of the Skin Irritant Potential of CS Aerosols on Human Skin Under Tropical Climatic Conditions." Edgewood Arsenal Technical Report, EATR 4252.

Punte CL, Owens EJ, Gutentag PJ. "Exposures to Orthochlorobenzylidine Malononitrile; Controlled Human Exposures." Arch of Environmental Health, Vol 6;72-80, 1963.

Shumes E, Taylor JS. "Industrial Contact Dermatitis: Effects of the Riot Control Agent Orthochlorobenzylidene Malononitrile." Arch Dermatol, Vol 107, Feb 1973.

Rengstorff RH. "Tear Gas and riot Control Agents: A Review of Eye Effects." The Optometric Weekly, Sept 1969.

Report of the Enquiry into the Medical and Toxicological Aspects of CS, Part II. Her Majesty's Stationery Office, London, Sept 1971.

Stein AA, Kirwan WE. "Chloracetophenone (Tear Gas) Poisoning: A Clinico-Pathologic Report." Current Topics, Vol 9, 3:374-3822.

Weigand DA. "Cutaneous Reaction to the Riot Control Agent CS." Military Medicine, pp 437-440. 